\title{
A PROFITABILITY COMPARISON BETWEEN A "ONE-WAY" CAR SHARING SERVICE AND A "MODIFIED ONE-WAY" CAR SHARING SERVICE
}

\author{
Massimo Fantola ${ }^{1}$, Alessandro Olivo ${ }^{2}$, Roberto Devoto ${ }^{3}$, Nuria Diaz-Maroto Llorente ${ }^{4}$ \\ 1,2,3 Department of Civil Engineering, Environment and Architecture, University of Cagliari, Italy \\ ${ }^{4}$ Department of Mathematics and Computer Science, University of Cagliari, Italy
}

Received 26 October 2016; accepted 15 January 2017

\begin{abstract}
The research looks at comparing the different performance offered by two types of one-way car sharing services. In particular, we compare the "traditional" service in which users can return vehicles to a pre-determined permitted parking space to the "modified" service in which the decision of where to return the vehicle to is made at the end of its usage and vehicles can be returned also outside the permitted parking areas. The comparison is based on common and given demand/offer assumptions. The mathematical modelling uses state of the art algorithms that allow us to determine for both types of service the optimal number of personnel to re-position the vehicles in order to maximise profit. In particular, the attractiveness of the two services herewith compared, has been analysed both in terms of overall profitability as well as in terms of maximum number of users. The results show and quantify how the "modified" service, whilst allowing a greater degree of flexibility to users in terms of return locations, causes lower economic returns for the service company and lowers the number of users that can be served. Finally, the model allows us to calculate the required tariff increase necessary to transform a "traditional" service into a "modified" service assuming an inelastic demand curve as well as constant profits for the service company.
\end{abstract}

Keywords: One-way carsharing; modified one way car sharing; relocation; staff sizing; optimization; integer programming.

\section{Introduction}

Car sharing is a service carried out by specialized companies that offers customers the opportunity to use a vehicle through the purchase of a ticket corresponding to the required use. It is a commercial activity, supported by sustainable mobility policies, which facilitates the transition from possession of a vehicle to the simple use of it, allowing customers to give up their private car whilst still having the flexibility to travel according to their needs. The car is therefore transformed from an expense to a service. (Shaheen and Cohen, 2007; Jorge and Correia, 2013).

The worldwide evolution of car sharing is plain to see; for explanatory purposes, it can be broken down into three essential phases: "traditional car sharing", "one-way car sharing" and "Car2Go".

\footnotetext{
${ }^{2}$ Corresponding author email: olivo@unica.it
} 
Traditional car sharing allows the customer to book the use of a vehicle, to be collected at a pre-arranged time and from a specified pick-up station. The customer is then required to return the vehicle to the same station after the agreed period of time.

A more attractive and flexible service is "oneway car sharing", which differs from the traditional method insofar as the customer may select different pick-up and dropoff stations during the booking process (Nourinejad and Roorda, 2014). One-way car sharing may also offer the user an extra option: instead of selecting the drop-off station while making the booking, the user may choose between various stations, within a determined perimeter, during their use of the vehicle. This type of service will be referred to as "modified one-way car sharing".

Finally, large urban areas have seen the development of free-floating car sharing services, such as "car sharing 2go". As part of such services, cars can be picked up directly by the user without any booking or time constraints, from any of the service's pickup stations within the urban area (Ciari et al., 2014).

Small and medium-sized urban areas, on which this study will focus, are usually provided with services based on a booking system, and are therefore attributable to traditional, one-way and modified one-way car sharing.

The desirability of each type of car sharing varies with regard to their operational characteristics and depends, first and foremost, upon the ease with which the user is guaranteed vehicle availability, both in the desired car park and at the desired time. This request is often difficult to guarantee, as it would generally create a surplus of vehicles at pick-up stations in areas where demand is low, and a lack of vehicles in areas of high demand (Weikl and Bogenberger, 2013).

The need to overcome this criticism has led to the adoption of various running methods, aimed at finding a balance between demand and availability.

- The first is based on the delegation of this task to the users (Uesugi, et al., 2007; Barth et al., 2004; de Almeida Correia et al., 2013; Clemente et al., 2013; Di Febbraro et al., 2012), providing them with a variable tariff with regard to the station or area to which they intend to return the vehicle. This system penalizes those users who wish to travel to a destination where demand is proven to be low. Evidently, the use of such a system may result in the cancellation of the request.

- The second focuses on selection between pre-determined bookings; in other words, only those booking wishing to return the vehicle to stations or areas of high demand are accepted, and other, less profitable bookings are refused (Fan et al., 2008; Correia and Antunes, 2012; Jorge et al., 2012). Consequentially, not all requests can be satisfied using this system. It is proven that, under these conditions, the overall level of service provided to customers declines.

- The third is based on the use of staff to bring vehicles from stations or areas where there is a surplus to pick-up stations where a request could otherwise not be satisfied. Evidently, when using this system, the business must take into account the extra costs incurred through employing staff to relocate vehicles. 
The desirability of car sharing services based on a booking system also depends on the level of freedom offered to the customer at the time of booking, in terms of the vehicle drop-off times and places available. With particular regard to the vehicle drop-off station, there is a growing trend in desirability, developing in the following manner:

- from the "traditional" service, which is completely fixed in terms of pick-up and drop-off station;

- to the "one-way" service, which allows the user some freedom when choosing the desired stations, but does still obligate the client to choose them at the time of booking;

- to the "modified one-way" service, in which the user is free, at the drop-off time, to leave the vehicle at any legally available station within the area covered by the service.

The increase in desirability for the user brings with it new criticisms, some of which concern the company and others of which concern the use itself. The growing ease with which a client is able to access the service and the flexibility offered to them in terms of returning the vehicle, are chiefly accompanied by a reduction in the number of customers served, due to the longer times required for relocation. They are also characterized by an increase in managerial costs, which is principally due to the higher number of staff employed; staffing costs, when compared with tariffs, produce a decrease in company revenue.

For this reason, choosing the best setup for a car sharing system is neither easy nor immediately obvious. It would appear that a more in-depth study is required in order to investigate which criteria, both for the company and the user, produce a car sharing service that guarantees more freedom for its users.

\section{Objectives and Work Methodology}

This study aims to provide a detailed comparison between two car sharing services: the "one-way" system and the "modified one-way" system. The two systems will be analyzed in the same reference context in order to determine their desirability for the user, the profits gained and the overall level of demand satisfied.

For this purpose, both systems are assumed to run in the same medium-sized urban environment. More specifically, the average dimensions of the domain replicate an area of interest whose diameter it is possible to cross in an average time of thirty minutes, in prevalent traffic conditions. Both of the services being compared are based on a first-come, first-served booking system and use the same supply system in terms of number, location and capacity of stations, and in the number of available vehicles. It is also assumed that both services receive the same level of demand, to be examined and applied over a determined length of time.

To combat the imbalance that exists between supply and demand in both systems, the model has been designed to include a vehicle relocation service, carried out by staff members. As part of this service, operators travel to the vehicles they must collect using collapsible motorcycles, which they then place inside the car during its transfer.

Taking into account the running costs of the vehicles and motorcycles, the distances between the stations, the staffing costs and the service tariff, a model based on 
maximizing the profits earned by the managing company and the number of requests fulfilled may be used to determine the optimal configuration of the vehicle relocation service for each of the two systems. This, in turn, allows us to investigate the extent to which the greater freedom granted to the user, intended as the waiving of the requirement to choose the drop-off station during the booking process, results in a loss of earnings for the company and in a reduced quality of service to customers as a whole.

Finally, from the context of a fixed demand and the constraints of the iso-revenue, it is possible to calculate how much the vehicle rental tariff must be increased in order to provide a service that allows the user this degree of freedom, whilst continually maintaining the profit levels earned by the one-way service.

In order to reach these objectives, a work methodology, composed of an analysis for each of the two different systems, has been created.

\subsection{One-way Car Sharing}

In this system, customers may enjoy the use of a vehicle if they adhere to four conditions, which are agreed during the booking procedure:

- The station from which the vehicle must be picked up at the start of the journey;

- The time at which the vehicle may be picked up at the start of the journey;

- The station at which the vehicle must be dropped off at the end of the journey;

- The time at which the vehicle must be dropped off at the end of the journey.
Under these conditions, the number of vehicles on offer at a given time and station is equal to the number of vehicles present at that same time and station, whether they are waiting to fulfill a future booking or waiting to be transferred to another station.

The demand in a given station corresponds to the number of vehicles that the users wish to pick up from that same station in a given moment. This demand may be fulfilled using the vehicles present in the station at the time, or, thanks to the transfer service, using vehicles that have been left in other stations, where they have not been requested for use.

The transfer service, carried out solely by staff using collapsible motorcycles, can be described as follows:

- One trip, made by the operator on the motorcycle provided, from the current station to the station in which the required car must be dropped off. In this case, the journey length is predetermined, as is the time taken to complete it.

- One trip, made by the operator, this time driving the vehicle with the motorcycle inside, from the station in which the car was parked to the station at which it has been requested. In this scenario, as before, the distance travelled by the operator and the average time taken to make the journey have been precalculated.

All of the vehicles made available to users in both of the two systems are fitted with a GPS system that tracks their movements and position at any given time. 
With regard to the booking system, each time a new booking arrives, the value of the demand at the desired pick-up station increases by one unit. Should the demand exceed the number of parking spaces present, the booking will be refused.

In the opposite case, the manager considers relocating vehicles and checks whether:

- The vehicles can be delivered to meet the requested time and station;

- The space required by the transferred vehicle is available in the drop-off station, at the established time.

Where both of these requirements can be satisfied, the booking is accepted; otherwise, it is refused.

An optimal relocation plan tends to function so as to ensure that each station contains the maximum number of available and usable vehicles, the costs incurred by operators carrying out transfers are reduced to a minimum, and the highest possible number of booking requests is fulfilled.

The results of the trial period, obtained by applying the model to the context of one-way car sharing, will show, respectively, the rate of satisfied requests and the corresponding profit level obtained. The model aims to use the minimum number of operators and transfers required to guarantee the maximum possible profitability of the system.

\subsection{Modified One-way Car Sharing}

In this system, customers may enjoy the use of a vehicle by respecting only three conditions, which are agreed during the booking process:
- The station from which the vehicle may be picked up at the start of the journey;

- The time at which the vehicle may be picked up at the start of the journey;

- The time at which the vehicle must be dropped off at the end of the journey.

The absence of any restrictions regarding the selection of a specific drop-off station, to which the car must be returned, introduces another limitation to the system management: in order not to run the risk of there being no available parking spaces at the relocation stations used by the operators, customers are not permitted to drop the cars off at these points. As a consequence, each vehicle returned by a client must then be relocated.

The previously stated considerations regarding supply and demand at the pickup stations remain valid for this scenario.

As regards the transfer of vehicles, in this case:

- The distance and time taken by operators to travel from a station to the location, indicated by GPS, in which a car has been dropped off cannot be determined in advance;

- The distance and time taken to move the vehicle from the location in which it has been parked to the station at which it is required cannot be determined in advance.

To overcome the impossibility of calculating such quantities, a fictitious node has been created (representing a drop-off station), to which vehicles are returned at the end of a rental period. The capacity of this fictitious station is equal to the total number of vehicles 
and its location is equidistant to all other stations. In terms of travel time, its distance from the other stations is equal to the average diameter of the urban area covered by the car sharing service (30 minutes).

This last condition may seem redundant, but is necessary to ensure that the system does not enter into crisis, and to provide the model with the information necessary for it to function. In fact, at the end of a rental period, the optimization program allocates the most convenient station for relocation, based on the incoming bookings. The overall times and costs allocated by the system are equal to or greater than those that would be incurred in reality.

The conditions concerning the acquisition of bookings and the relocation of vehicles remain the same.

\section{Time-space Reference Graph}

The management of the described system can be modeled in a $\mathrm{G}(\mathrm{N}$; A) space/time graph, where all of the nodes under $\mathrm{N}$ represent the stations for each time period shown across the horizontal axis of the graph. For example, Figure 1 shows a network in which the nodes form a circular shape. They represent four stations, which are labeled using the letters $\mathrm{A}$ to $\mathrm{D}$ and are replicated for six time periods, labeled with the numbers 1 to 6 . The sequence of activities carried out for each operator is represented by a route in $\mathrm{G}(\mathrm{N} ; \mathrm{A})$.

The lines between a station in a period to the same station in the following period represent a static activity carried out by an operator, who either remains at the station between these periods waiting to carry out a dynamic activity (relocating a vehicle or reaching a vehicle that must be collected and relocated), or is carrying out a routine maintenance activity in the station between these periods. The waiting and maintenance times are represented in Figure 1 by broken lines with dashes of equal length, and by dotted lines, respectively. For example, in Figure 1, an operator waits in station B between periods 1 and 2 and carries out maintenance in station $\mathrm{D}$ between periods 3 and 4.

The lines running from one station in one time period to a different station in another time period represent operators moving between stations using a motorcycle or car. Motorcycle or car journeys are represented in Figure 1 by broken lines with dashes of varied length and by continuous lines, respectively. Figure 1 shows an operator travelling by motorcycle from station $\mathrm{D}$ in period 4 to station $\mathrm{A}$ in period 5 , and then to station $\mathrm{C}$ in period 6. All of the movements in which an operator drives a car foresee a motorcycle on board that same car.

Determining the sequence of activities carried out by personnel is not sufficient to resolve the problem of acceptance or even of a booking. A number of factors must be pre-determined, including the number of available and unavailable vehicles stored in each station, how many vehicles will not be allocated to customers due to a lack of available vehicles, and how many vehicles may not be dropped off at the desired station due to a lack of free spaces. Evidently, in scenario II, the latter problem does not exist; vehicle drop-off is always possible insofar as the client may leave the vehicle in any of the legally available stations owned by the company within the urban area. 


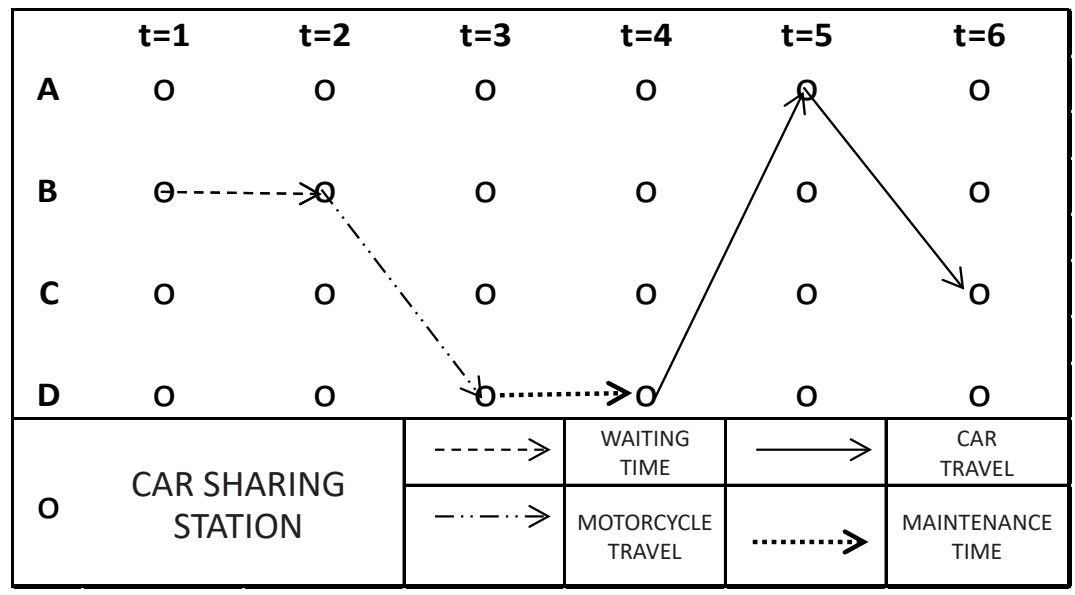

Fig. 1.

Time-Space Network for Planning Car Relocation

\section{The Optimization Model}

I represents the total number of stations $T$ represents the sum of the continuous time periods that form the horizontal axis of the graph. The index of period $t \in T$, has a value between 1 and $|\mathrm{T}|$. it $\in N$ is the node that represents station $i \in I$ in period $t \in T$. Ay is the total time spent waiting, $\mathrm{Az}$ is the total time spent on maintenance, $\mathrm{Au}$ is the total time that operators spend travelling by motorcycle and Av is the total time taken by operators to travel by car for relocation purposes. $\mathrm{K}$ is the total number of operators that can be carrying out waiting and maintenance activities, or travelling by car and motorcycle for relocation purposes. dit is the number of cars required in station $i \in I$, between periods $\mathrm{t}-1 \in \mathrm{T}$ and $t \in T$, and sit is the number of cars returned to station $i \in I$ between periods $\mathrm{t}-1 \in \mathrm{T}$ and $t \in T$.

pi indicates the maximum number of vehicles that can be stored in a generic station $i \in I$ . From the moment in which each vehicle must be removed from or parked in an available parking space, dit and sit may not be greater than pi. $\tau$ is the time required to complete a routine maintenance activity on one of the system's vehicles, and mit is the number of vehicles dropped off in station $i \in I$ between periods $\mathrm{t}-1 \in \mathrm{T}$ and $t \in T$ that require maintenance. Therefore, mit $<$ sit.

The principle deciding variables are:

- The variable $y^{k}{ }_{i t, i t+1}$ has a value of 1 if the operator $k \in K$ waits at station $i \in I$, between periods $t \in T$ and $t+1 \in T$; otherwise, it has a value of 0 .

- The variable $\mathrm{z}^{k}{ }_{i, i t+1}$ has a value of 1 if the operator $k \in K$ carries out maintenance on a vehicle at station $i \in I$ between periods $t \in T$ and $t+1 \in T$; otherwise, it has a value of 0 .

- The variable $u^{k}{ }_{i t, j t+t i j}$ has a value of 1 if the operator $k \in K$ travels by motorcycle from station $i \in I$ in period $t \in T$ to station $j \in I$ in period $t+t i j \in T$ 
; otherwise, it has a value of $0 . \mathrm{c}^{u k}{ }_{i t, j t+t i j}$ represents the relative cost per unit.

- The variable $\mathbf{v}_{i t, j+t i j}^{k}$ has a value of 1 if the operator $k \in K$ drives a car from station $i \in I$ in period $t \in T$ to station $j \in I$ in period $t+t i j \in T$; otherwise, it has a value of $0 . c^{v k}{ }_{i t, j t+t i j}$ represents the relative cost per unit.

- The variable $\mathrm{x}_{\mathrm{it}}^{\mathrm{a}}$ represents the number of vehicles available at station $i \in I$, at the start of period $t \in T$.

- The variable $\mathrm{x}^{\mathrm{n}}{ }_{\mathrm{it}}$ represents the number of vehicles that are not available, but which are kept at station $i \in I$ at the start of period $t \in T$. $\mathrm{c}^{\mathrm{n}}$ is the cost per unit relative to the loss incurred due to storage of unavailable vehicles. To render them available, a maintenance procedure must be carried out.

- The variable $\mathrm{x}^{\mathrm{d}}{ }_{\mathrm{it}}$ represents the number of vehicles that cannot be made available to the users at station $i \in I$, between periods $t-1 \in T$ and $t \in T$, due to a lack of available vehicles and / or lack of personnel available to relocate vehicles. $\mathrm{c}^{\mathrm{d}}$ is the cost per unit corresponding to the loss incurred due to a lack of vehicles.

- The variable $\mathrm{x}_{\mathrm{it}}^{\mathrm{s}}$ represents the number of vehicles that are unavailable for use at station $i \in I$ between periods $t-1 \in T$ and $t \in T$, due to a lack of available space and / or personnel to relocate vehicles. $\mathrm{c}^{\mathrm{s}}$ is the cost per unit corresponding to the loss incurred due to saturation of the stations.

An operator $k \in K$ may only carry out one activity in the first period. This restriction is formalized as follows:

$$
\begin{aligned}
& \sum_{(i 1, i 2) \in A y} \quad y^{k}{ }_{i 1, i 2}+\sum_{(i 1, i 1+\tau) \in A z} \\
& \mathrm{z}^{k}{ }_{i 1, i 1+\tau}+\sum_{(i 1, j 1+t i j) \in A u} \quad u^{k}{ }_{i 1, j 1+t i j}+
\end{aligned}
$$

$$
\sum_{(i 1, j 1+t i j) \in A v} \quad \mathrm{v}^{k}{ }_{i 1,1 t+t i j}=1
$$

$\forall k \in K(1)$

The continuity of the activity for each operator is $k \in K$, and for each node is $i_{t} \in N$, so that $\mathrm{t} \neq 1$ and $\mathrm{t} \in|\mathrm{T}|$ can be expressed as follows:

$$
\begin{aligned}
& y_{i t-1, i t}^{k}+\mathrm{z}_{i t-\tau, i t}^{k}+\sum_{j t-t j, i \in N} u_{j t-t i j, i, t}^{k}+ \\
& \sum_{j t-t j, i \in N} v_{j t-t i j, i, t}^{k}=y_{i t, i t+1}^{k}+\mathrm{z}^{k}{ }_{i t, j i t+\tau}+ \\
& \sum_{j t+t i, j \in N} \\
& v_{i t, j t+t i, j}^{k} \\
& u^{k}{ }_{i t, j t+t i, j}+\sum_{(j t+t i, j \in N}
\end{aligned}
$$$$
\forall i t \in N, t=2, \ldots,|\mathrm{T}|-1, \forall k \in K(2)
$$

An operator $k \in K$ may only carry out one activity in the final period. This restriction is formalized as follows:

$$
\begin{array}{lc}
\sum_{(i|\mathrm{~T}|-1, i|\mathrm{~T}|) \in A y} & y^{k}{ }_{i|\mathrm{~T}|-1, i|\mathrm{~T}|}+ \\
\sum_{(i|\mathrm{~T}|-1, i|\mathrm{~T}|) \in A z} & \mathrm{z}^{k}{ }_{i|\mathrm{~T}|-\tau, i|\mathrm{~T}|}+ \\
\sum_{(i|\mathrm{~T}|-t i, j ; j|\mathrm{~T}|) \in A u} & \mathrm{u}^{k}{ }_{i|\mathrm{~T}|-\mathrm{ti}, \mathrm{j} ; \mathrm{j}|\mathrm{T}|}+ \\
\sum_{(i|\mathrm{~T}|-t i, j ; j|\mathrm{~T}|) \in A v} & \mathrm{v}^{k}{ }^{k}|\mathrm{~T}|-\mathrm{ti}, \mathrm{j} j|\mathrm{~T}| \\
& \forall k \in K(3)
\end{array}
$$

The number of available vehicles at each station and in each time period is updated as follows:

$$
\begin{aligned}
& \mathrm{x}^{\mathrm{a}}{ }_{\mathrm{it}}=\mathrm{x}^{\mathrm{a}}{ }_{\mathrm{it}-1}+\sum_{k \in K}\left(\sum_{j t-t j, i \in N} \quad \mathrm{v}^{k}{ }_{i t-t j i, j t}+\right. \\
& \left.\mathrm{z}^{\mathrm{k}}{ }_{i t-\tau, i t}-\sum_{j t+t i, j \in N} \quad \mathrm{v}^{k}{ }_{i t, j+t+i j}\right)+s_{i t}-\mathrm{x}^{\mathrm{s}}{ }_{\mathrm{it}}-d_{i t}+ \\
& \mathrm{x}^{\mathrm{d}}{ }_{\mathrm{it}}-m_{i t}
\end{aligned}
$$


The number of unavailable vehicles at each station and in each time period is updated as follows:

$\mathrm{x}^{\mathrm{n}}{ }_{\mathrm{it}}=\mathrm{x}_{\mathrm{it}-1}^{\mathrm{n}}-\sum_{k \in K} \quad \mathrm{z}^{k}{ }_{i t-1, i t-1+\tau}+m_{i t}$

$$
\forall i \in I, \forall t \in T
$$

The number of available and unavailable vehicles at a station may not exceed the capacity of that station $i \in I$ between periods $t-1 \in T$ and $t \in T$. Therefore:

$\mathrm{x}^{\mathrm{a}}{ }_{\mathrm{it}}+\mathrm{x}^{\mathrm{n}}{ }_{\mathrm{it}}+s_{i t+1}-\mathrm{x}_{\mathrm{it}+1}^{\mathrm{s}} \leq p_{i}$

$\forall i \in I, \forall t \in T(6)$

The number of unfulfilled returns must be lower than the total number of returns planned for each node, in each period. Therefore:

$\mathrm{x}_{\mathrm{it}}^{\mathrm{s}} \leq \mathrm{s}_{\mathrm{it}}$

$\forall i \in I, \forall t \in T(7)$

The number of unfulfilled requests must be lower than the total number of bookings planned for each node, in each period. Therefore: $\mathrm{x}^{\mathrm{d}}{ }_{\mathrm{it}} \leq \mathrm{d}_{\text {it }}$

$\forall i \in I, \forall t \in T$ (8)

The transport costs for motorcycles and cars, as well as the costs incurred due to lack of vehicles and saturation of the stations, are minimized through:

$$
\begin{aligned}
& \mathrm{z}=\min \sum_{k \in K} \sum_{i t \in N} \sum_{j t+t i j \in N} \quad\left(\mathrm{c}^{u k}{ }_{i t, j t+t i j}\right. \\
& \left.* u^{k}{ }_{i t, j t+t i j}+\mathrm{c}^{\mathrm{vk}}{ }_{i t, j t+1}{ }^{*} \mathrm{v}^{k}{ }_{i t, j t+t i j}\right)+\mathrm{c}^{\mathrm{d} *} \sum_{i t \in N} \quad \mathrm{x}^{\mathrm{d}}{ }_{i \mathrm{t}} \\
& +\mathrm{c}^{\mathrm{s}} * \sum_{i t \in N} \quad \mathrm{x}_{\mathrm{it}}^{\mathrm{s}}+\mathrm{c}^{\mathrm{n} *} \sum_{i t \in N} \quad \mathrm{x}^{\mathrm{n}}{ }_{i t}
\end{aligned}
$$

\section{Trial Period}

In the "one-way" system, each booking is characterized by four attributes: the pick-up station, the drop-off station, the rental start time and the rental end time. However, the "modified one-way" system is characterized by three such attributes: the pick-up station, the rental start time and the rental end time.

Using an increasing number of operators $(0,1,2,3, \ldots, n)$ for relocation operations, the results gained through using the two systems may be compared. With a specified number of operators, the proposed model is executed for the first time using the data from the first booking, and continues so that with the arrival of the nth booking, it will be executed using the data collected from all $\mathrm{n}$ bookings received hereto. With each new instance to process, two conditions must be verified: whether the supply and demand values are lower than the station capacity, and whether the number of vehicles necessary to serve all customers is lower than the number of vehicles available at the time of the last solution proposed by the model. Where these conditions are not met, the booking is automatically refused without launching any optimization. Where this is not the case, an instance is generated and resolved using a mathematical problemsolver. In the trial period, the solver is Ilog Cplex Optimization Studio 12.5, which uses state-of-the-art algorithms to solve mixed programming problems. The trials were executed on a laptop with $2.5 \mathrm{Ghz}$ and 8 $\mathrm{Gb}$, using predefined parameter settings. The proposed model is executed for five minutes. If, in this time interval, all of the variables $x d i t$ e xsit assume the value 0 in the solution proposed by the model, the latest booking is accepted. In the opposite case, the 
booking is refused, either because at least one user would not find an available car at their chosen pick-up station, or due to saturation at the drop-off station preventing a user from returning the vehicle to their chosen station (evidently, the latter condition refers only to Scenario I).

More formally, where $\mathrm{B}$ is the total number of bookings and $\mathrm{Cb}$ is the minimum number of vehicles to be assigned to users after the arrival of the generic booking $b \in$ $\mathrm{B}$, given that each booking requires only one vehicle, $\mathrm{Cb}$ may be calculated as the maximum number of bookings that can be fulfilled across all of the time periods. In such conditions, it follows that:

$$
C_{b}=\max _{t \in T}\left(\sum_{j=1}^{b} \delta j t-\sum_{j=1}^{b-1} \gamma j\right),
$$

where $\delta \mathrm{jt}=1$, a generic booking $\mathrm{j} \in \mathrm{B}$ requesting the use of a vehicle in period $t \in$ $T$ is fulfilled using a vehicle available at time $t \in \mathrm{T}$; otherwise, it has a value of $0 . \gamma j=1$ if a booking $j \in B$ that arrived before $b \in B$ is fulfilled; otherwise, its value is 0 .

If the booking is rejected, and $\mathrm{Cb}$ is lower than the total number of vehicles available, this means that, for one of the bookings, although there is an available vehicle in the system at the time of the request, there is no operator available to relocate that vehicle from its current location (drop-off station or fictitious station) to the required pick-up station before the booking start time.

The trial period was carried out using a network of 30 cars and 30 stations, labeled with the letters A to D2, each of which is able to store up to two cars. Scenario II uses an additional, fictitious station, labeled E2, which has a capacity of 30 cars and represents all of the vehicle drop-off parking spaces available in Scenario II. Given that, in Scenario II, it is impossible to predetermine the drop-off point chosen by a user to return a vehicle, all returns are hypothetically carried out using the fictitious station E2. This station is assumed to be equidistant from each of the real stations in terms of travel time; as a precaution, this distance has been set to 30 minutes' travel times.

At the start of the planned time, there is one car in each of the thirty pick-up stations. The continuous time periods shown in the graph are indicated by $\mathrm{T} ; 48$ periods of time, each one ten minutes in length, are represented in the graph across a total time span of eight hours.

The unitary costs used in the study are as follows:

- Motorcycle travel per kilometre: $0.08 €$;

- Car travel per kilometre: 0.12€;

- Penalty for unfulfilled booking $\left(c^{d}\right)$ : $500 €$;

- Penalty for inability to return a car due to saturation $\left(c^{s}\right): 400 €$;

- Penalty for storage of unavailable vehicles $\left(c^{n}\right): 300 €$.

In scenario II, given that cars may be returned to any parking point, the penalty for inability to return a car due to saturation in not considered; as such, cost cs remains at zero. Furthermore, for obvious needs of simplification, this study has not considered the need to carry out maintenance procedures on vehicles in either of the two scenarios executed in the trial period.

The results analysis for both of the two scenarios (shown in Tables $1,1 b, 2 a$ and $2 b)$ firstly demonstrate how an increase in the number of operators employed to 
collect cars, thereby reducing the transfer costs, contributes to a higher rate of demand fulfillment, where the maximum time given to the Cplex solver to arrive at a solution remains at 5 minutes. Moreover, comparison of the results gained through responding to demand in scenarios I and II demonstrates that the introduction of the freedom to choose the drop-off point reduces the rate of demand fulfillment and increases relocation costs. The time limit for the Ilog Cplex Optimization Studio to resolve the optimization instances remains the same.

In the Table, each row is associated with one booking, within which the columns indicate the corresponding pick-up station (DS), the rental start time (DP), the drop-off station (AS), and the rental end time (AP). The column marked "Rev." indicates the revenue produced by each booking.

The proposed model is executed, in the two scenarios, according to four possible dimensional configurations:

- no operators, shown as $|\mathrm{K}|=0$ (valid only in scenario I).

- $\quad$ one operator, shown as $|\mathrm{K}|=1$;

- two operators, shown as $|\mathrm{K}|=2$;

- three operators, shown as $|\mathrm{K}|=3$,
- $\quad$ four operators, shown as $|\mathrm{K}|=4$.

Configuration $|\mathrm{K}|=0$ has not been used in Scenario II as the proposed model is restricted to using at least one operator for vehicle transfer. This is due to the fact that, in this scenario, the vehicle return time is pre-arranged, but the location is not.

For each configuration, where $\mathrm{N}$ is the number of instances processed and satisfied, the results obtained are as follows: $\mathrm{Cb}$ is the minimum number of cars to be assigned to a user before processing a generic booking $b$ $\in B$. The minimum-cost objective function in the proposed model is $\mathrm{z}[€]$. The optimal solution gap, Gap[\%], represents the percentage deviation of the solution found in optimal conditions. All of the resolved instances have demonstrated a good level of optimization, as can be seen by analyzing the corresponding gap values.

The cumulative revenue generated by the bookings fulfilled is labeled Tot Rev. The total cost (Tot. Cost) is calculated as the sum of the values corresponding to $\mathrm{z}$ and the fixed costs. The cumulative profit (Profit) relative to the bookings made is calculated as the difference between the cumulative revenue and the total costs. 


\section{Table 1a}

Result of One Way Car Sharing Testing

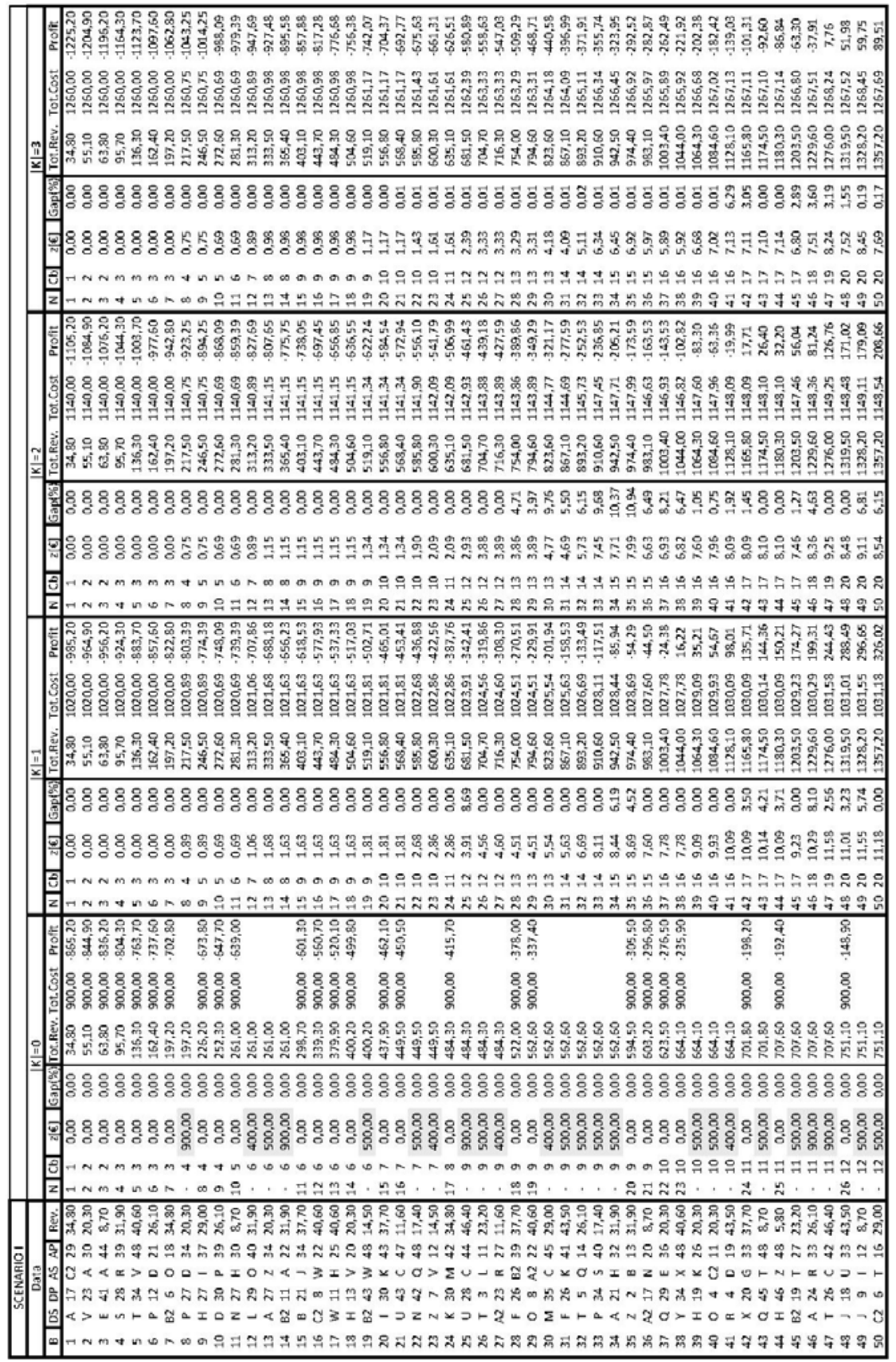




\section{Table 1b}

Result of One Way Car Sharing Testing

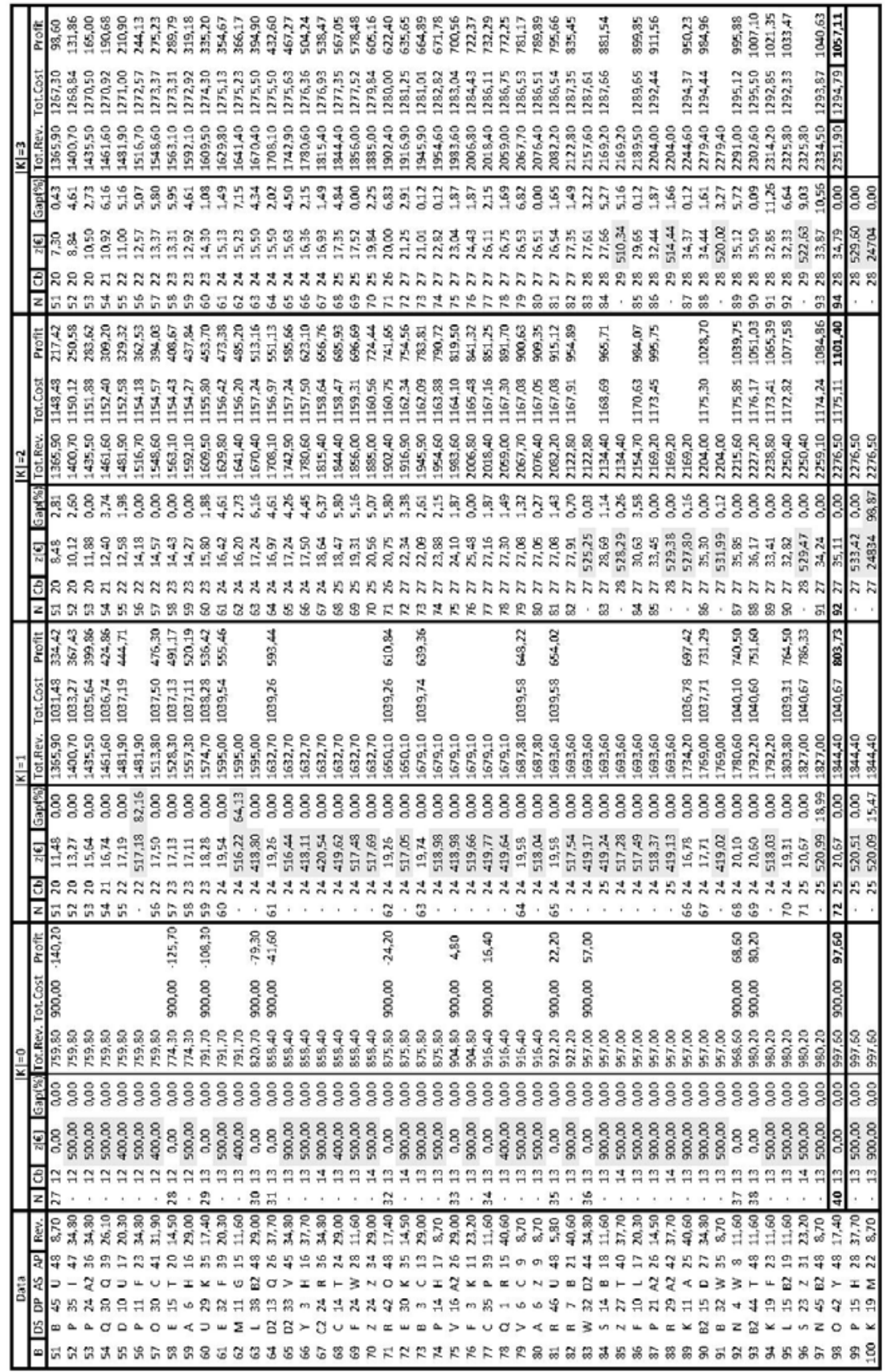




\section{Table 2a}

Result of Modified One Way Car Sharing Testing

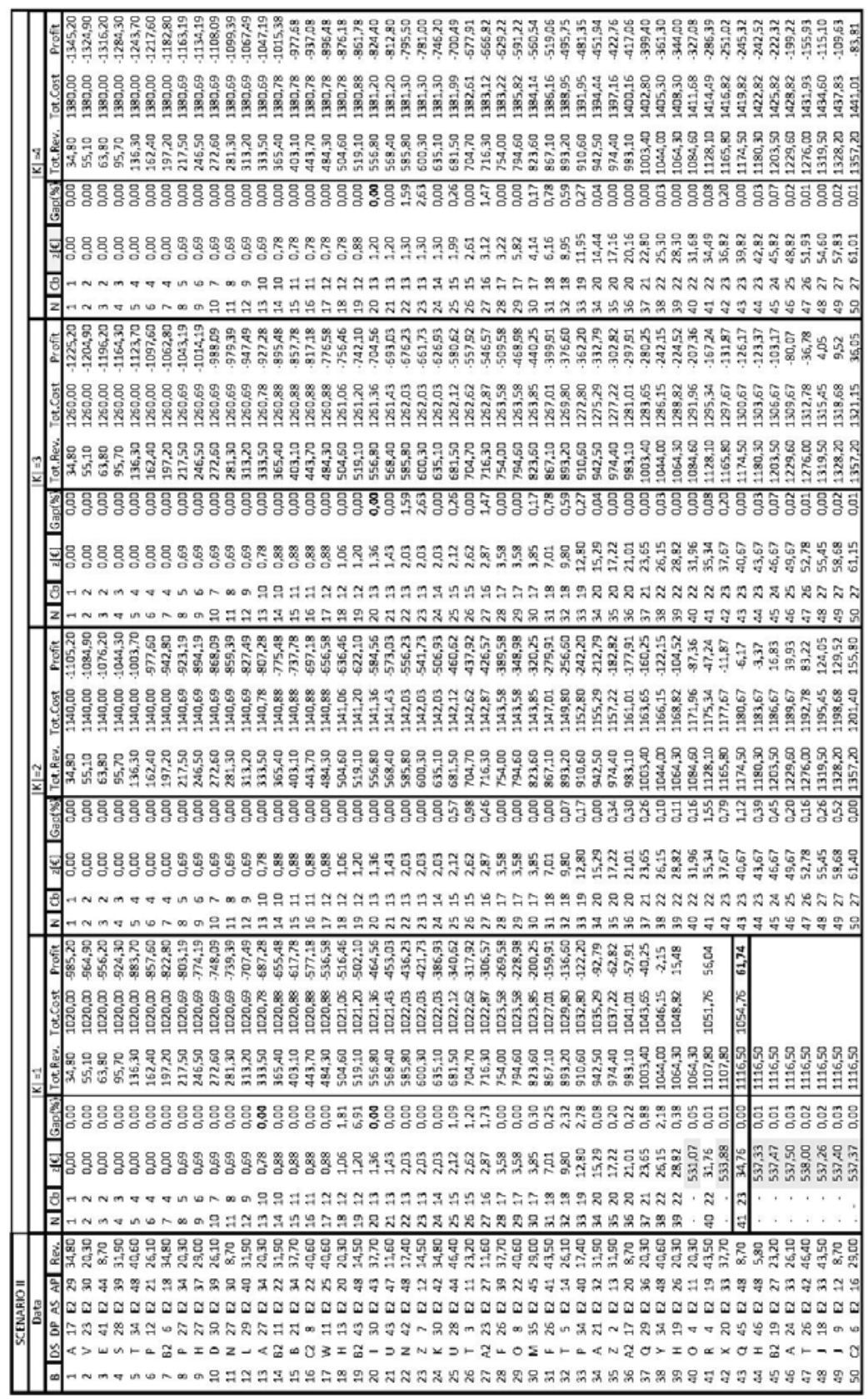




\section{Table 2b}

Result of Modified One Way Car Sharing Testing

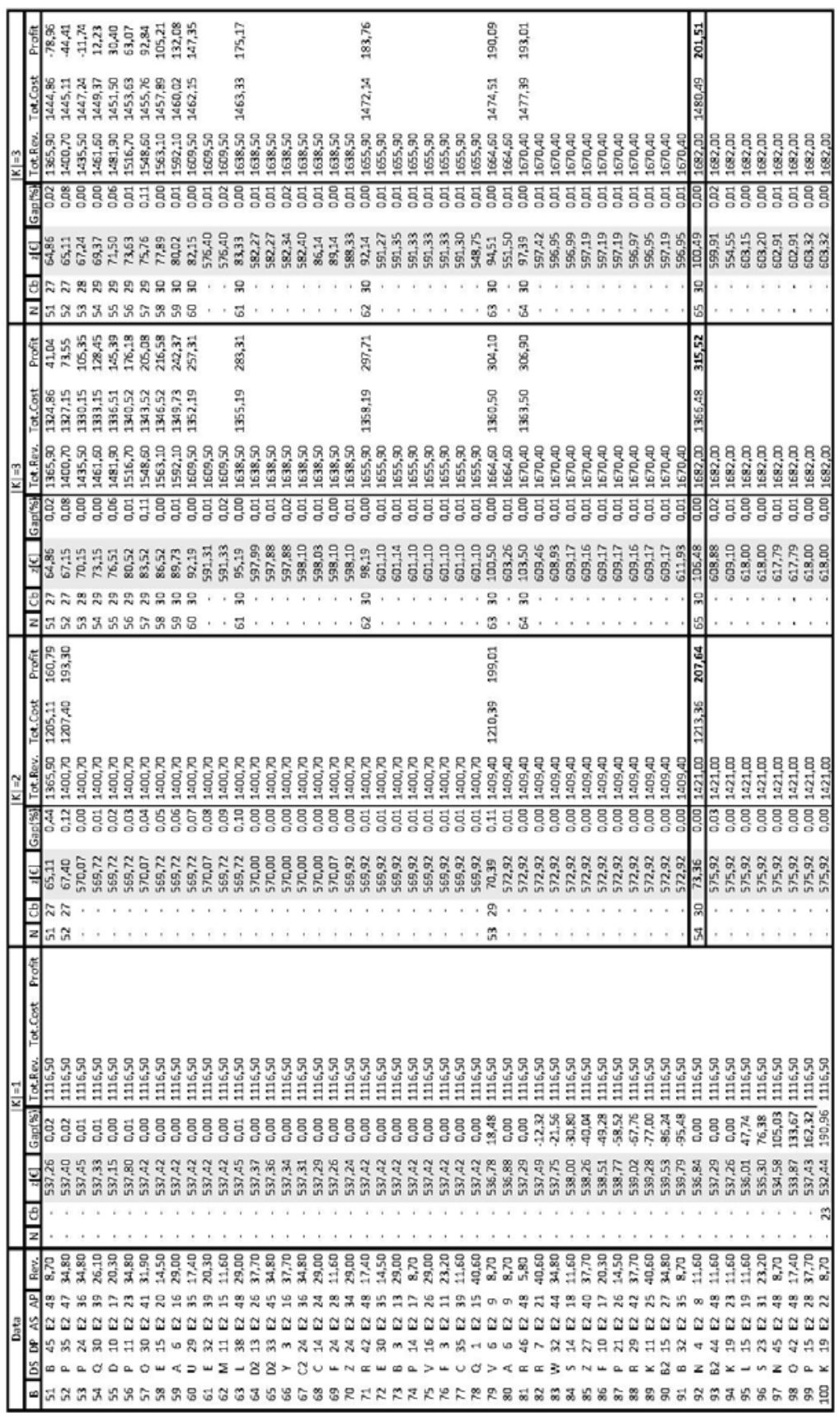


In this experiment, the fixed costs generated by the employment of operators for vehicle relocation have been calculated as $120 € /$ op for the eight-hour period considered. In addition to this are the costs of managing the cars on offer, calculated as $30 € / \mathrm{d}$ per car, the sum of the fixed costs + the running costs. It is assumed in the trial period that the distance covered by the car sharing vehicles each day is, on average, $80 \mathrm{~km}$.

The values shown in the tables indicate that in Scenario I (Tables $1 \mathrm{a}$ and $1 \mathrm{~b}$ ), the number of operators $(\mathrm{K})$ required to generate the highest level of profit (1101.40€), where 92 out of 100 incoming bookings were fulfilled, is two. In fact, although introducing a third operator $(\mathrm{K}=3)$ allows for a further two bookings to be fulfilled (94), the cumulative profit generated is lower, at $1057.11 €$. Evidently, this is mainly attributable to the economic burden of employing more than a certain number of operators on the flow of costs.

In Scenario II, the number of operators $(\mathrm{K})$ required to generate the highest profit (315.52€) is three. In this instance, the number of bookings fulfilled is 65 , out of a total of 100 booking requests processed. In fact, the use of 4 operators $(\mathrm{K}=4)$ allows for the same number of booking requests to be fulfilled (65), while reducing the profits to $201.51 €$.

It is worth noting that in Scenario II, the values representing the profits are certainly rounded down. In fact, the hypothesis that vehicle drop-off is carried out in a fictitious station at 30 minutes' distance from each of the real stations generates variable costs, corresponding to vehicle transfer and operators, whose values are certainly higher than the reality. This is due to the fact that such a hypothesis does not take into account any drop-off stations within the urban area covered by the service that are much closer to the departure and arrival stations.

\section{Conclusions}

This study provides a detailed comparison of the profitability of two car sharing systems: the "one-way and the "modified one-way" approaches, which can both be defined as evolutions of the first car sharing system created. In a "one-way" system, the user agrees the pick-up and drop-off stations and times with the company during the booking process, whereas a "modified oneway" system differentiates itself by allowing the user the freedom to choose the drop-off station up until the moment in which the vehicle must be returned, from a range of available locations within a given area.

The comparison of the two systems' profitability is based on various financial factors included in the services provided by both systems, within an identical supply system on a typical day, which displays an identical flow of bookings in the same space and time.

The two car sharing systems share the advantage of a relocation service, carried out by a team of operators. Following instructions sent from a control centre, these operators collect cars dropped off by users at the end of a booking, or unused cars sitting in one station, and move them to a different station where they have been requested by a client. The relocation procedure is carried out using collapsible motorcycles which are then placed inside the car during its transfer.

The optimization method uses a mathematical programming model capable of determining the minimum number of 
relocation operators required to maximize the company's profits. The trial has allowed some considerations to be formed.

The commercial activity carried out by the "one-way" car sharing system allows, in a best-case scenario, for a company profit of $1,101.40 €$, determined by a revenue stream of 2,276.50€ and a total expenditure (fixed costs + operating costs) of $1,175.11 €$. This best-case scenario requires the use of two staff members for relocation and fulfilled 92 out of 100 booking requests, distributed over a time period of eight hours. It is worth noting that only eight bookings were not fulfilled, due to the inability to guarantee an available vehicle or drop-off station.

On the other hand, the most profitable activity carried out by the "modified oneway" system required the use of three staff members for relocation, and allowed for 64 out of 100 bookings to be fulfilled. This activity generated a revenue stream of $1,682.00 €$, with a total expenditure (fixed costs + operating costs) of $1,366.48 €$ and a total profit of $315.52 €$.

The levels of profitability demonstrated in the latter of the two examples depend on numerous factors, the most important of which concerns the higher number of staff members required for relocation. This is due both to the uncertainty of the drop-off station and to the fact that each vehicle must be reallocated, as storing it in the available parking spaces creates the risk of there being insufficient space for any vehicles waiting to be picked up. The aforementioned increase in relocation time is also conditioned by a lack of available vehicles with which to fulfill booking requests. Furthermore, as has already been mentioned, the low profitability level of the "modified" system is strongly influenced by the introduction of a fictitious station, at a distance of 30 minutes from each of the pick-up and drop-off stations, without which it would not be possible to run the proposed system.

The disparity in the results obtained demonstrates that the increase in desirability created by inserting the possibility of dropping off the vehicle in any station, without the obligation to choose that station during the booking process, generates a marked decrease in the revenue stream, which in turn raises the question of whether or not it is viable to maintain such a service. This point remains valid even though the comparison penalizes, albeit minimally, the profitability of the "modified" system: the distance between the fictitious station and the other stations accounts for the maximum, or more probably, a larger overall transfer cost than would realistically be incurred.

To this end, a so-called "rebalancing tariff" can be calculated, which is necessary to maintain a stable flow of profit through "modified" car sharing where there is a fixed demand. In the present case the increase in the tariff has been determined by comparing the difference between the profits accumulated daily in the two scenarios (785.88 €/day) with the daily usage time of the cars included in Scenario II $(5,800$ $\min$ /day).

In such conditions, the rebalancing tariff is $0.135 € / \mathrm{min}$.

\section{References}

Barth, M.; Todd, M. 1999. Simulation model performance analysis of a multiple station shared vehicle system, Transportation Research part C: Emerging Technologies 7(4): 237-259. 
Barth, M.; Todd, M.; Xue, L. 2004. User-based vehicle relocation techniques for multiplestation shared-used vehicle systems, Transportation Research Board 80th Annual Meeting (04-4161), 1-16.

Cepolina, E.M.; Farina, A. 2012. A new shared vehicle system for urban areas, Transportation Research part C: Emerging Technologies 21(1): 230-24.

Ciari, F.; Bock, B.; Balmer, M. 2014. Modeling stationbased and free-floating carsharing demand: test case study for Berlin, Transportation Research Record 2416: 37-47.

Clemente, M.; Fanti, M.; Mangini, A.; Ukovich, W. 2013. The vehicle relocation problem in car sharing systems: Modeling and simulation in a petri net framework. In Proceedings of International Conference on Applications and Theory of Petri Nets and Concurrency, Springer Berlin Heidelberg, 250-269.

Correia, G.H.D.A.; Jorge, D.R.; Antunes, D.M. 2013. The added-value of accounting for users' flexibility and information on the potential of a station-based one-way carsharing system: An application in Lisbon, Portugal, Journal of Intelligent Transportation Systems 18(3): 299-308. DOI 10.1080/15472450.2013.836928.

de Almeida Correia, G.H.; Antunes, A.P. 2012. Optimization approach to depot location and trip selection in one-way carsharing systems, Transportation Research part E: Logistics and Transportation Review 48(1): 233-247.

Febbraro, A.; Sacco, N.; Saeednia, M. 2012. One-way carsharing solving the relocation problem, Transportation Research Record: Journal of the Transportation Research Board 2319: 113-120.

Fan, W.; Machemehl, R.; Lownes, N. 2008. Carsharing dynamic decision-making problem for vehicle allocation, Transportation Research Record: Journal of the Transportation Research Board 2063: 97-104.
Jorge, D.; Correia, G. 2013. Carsharing systems demand estimation and defined operations: a literature review, European Journal of Transport and Infrastructure Research 13(3): 201-220.

Jorge, D.; Correia, G.; Barnhart, C. 2012. Testing the validity of the MIP approach for locating carsharing stations in one-way systems, Procedia-Social and Behavioral Sciences 54(2012): 138-148.

Kek, A.; Cheu, R.; Chor, M. 2006. Relocation simulation model for multiple-station shared-use vehicle systems, Transportation Research Record: Journal of the Transportation Research Board 1986: 81-88.

Nair, R.; Miller-Hooks, E. 2011. Fleet management for vehicle sharing operations, Transportation Science 45(4): 524-540.

Nourinejad, M.; Roorda, M. 2014. A dynamic carsharing decision support system, Transportation Research part E: logistics and transportation review 66: 36-50.

Shaheen, S.; Cohen, A. 2007. Growth in worldwide carsharing an international comparison, Transportation Research Record: Journal of the Transportation Research Board 1992: 81-89.

Uesugi, K.; Mukai, N.; Watanabe, T. 2007. Optimization of vehicle assignment for car sharing system. In Proceedings of International Conference on Knowledge-Based and Intelligent Information and Engineering Systems, Springer Berlin Heidelberg, 1105-1111.

Weikl, S.; Bogenberger, K. 2013. Relocation strategies and algorithms for free-floating car sharing systems, IEEE Intelligent transportation systems magazine 5(4): $100-111$.

Pelksmans, L.; Debal, P. 2006. Comparison of onroad emissions with emissions measured on chassis dynamometer test cycles, Transportation Research Part D: Transport and Environment 11(4): 233-241. 